\title{
"ANTROPOMETRIA DA POSTURA SENTADA - A MEDIÇÃO DAS CINCO VARIÁVEIS DE POSICIONAMENTO"
}

\author{
LAERTE MATIAS
}

\section{RESUMO}

Utilizando um equipamento básico que permitiu a regulagem de vinco variáveis que são responsáveis pelo posicionamento de sujeitos em posturas sentadas, foi realizado um experimento que gerou dados relativos às melhores recomendações de posicionamento do sujeito, aplicáveis a situações da posição sentada. O experimento possibilitou ainda se estabelecer uma metodologia de levantamentos em outras regiões brasileiras, e o estabelecimento de parâmetros antropométricos próprios, mais voltados às características da população brasileira, em substituição aos dados estrangeiros utilizados como referencial de projetos de produtos e de estações de trabalho no Brasil. Os resultados indicam a possibilidade de realização de outros experimentos e a utilidade das informações obtidas.

\section{PALAVRAS-CHAVE: Engenharia humana; Antropometria; Espaço de trabalho. Desenho industrial; Engenharia industrial.}

\section{INTRODUÇÃO}

Considerando-se as condições de pesquisa no Brasil, bem como a falta de uma tradição de trabalhos de pesqui$\mathrm{sa}$, voltados ao conhecimento do homem brasileiro, existindo somente um levantamento a nível nacional, que determinou peso e altura da população brasileira ${ }^{2}$, nas diferentes regiões em que se divide o Brasil, se constata a importância de trabalhos que visem definir estas características.

A tempos procura-se conhecer algumas das variáveis de posicionamento do sujeito, para poder comparar com os dados estrangeiros normalmente utilizados em projetos de produtos, equipamentos e estações de trabalho no Brasil. Foi então necessário o desenvolvimento de um experimento que possibilitasse conhecer estas variáveis ${ }^{1}$.

\section{2 - CARACTERÍSTICAS BRASILEIRAS}

No Brasil, de acordo com o levantamento realizado pelo Instituto Brasileiro de Geografia e Estatística $\mathrm{IBGE}^{2}$, a população está distribuída em 7 (sete) diferentes regiões; estas populações tem características de peso e altura, específicas em cada uma delas. Este fato dificulta o estabelecimento de valores gerais, ou padrões antropométricos representativos de toda a população sem que seja feito um levantamento $\mathrm{cm}$ todas estas regiões.

A falta de dados sobre a população brasileira, cria problemas de utilização indisciiminada de dados estrangeiros $\mathrm{cm}$ projetos. 0 que é agravado pela importação de tecnologias $\mathrm{cm}$ projetos tipo "caixa-preta", onde nem ao menos se realiza um trabalho de adaptação aos valores antropométricos básicos da população brasileira.
Os trabalhos de COBLENTZ ${ }^{3}$, nos ensinam a inconveniência de utilização de normas e valores antropométricos estrangeiros na concepção de projetos relacionados aos homens em postos e estações de trabalho, gerando problemas de saúde $e$ operacionalidade aos usuários.

A generalização do uso de dados antropométricos $e ́$ contestada por IGNAZI ${ }^{4}$, que sugere que este procedimento só é possível quando os dados biométricos são iguais, ou mesmo muito próximos, o que não se verifica no Brasil, relacionados aos dados de outros países, e nem mesmo em termos regionais.

0 trabalho sugere recomendações de levantamento. onde são apresentadas as 5 (cinco) variáveis, a definição da amostra experimental, o tipo de equipamento a ser utilizado, as orientações ao sujeito durante o experimento e os resultados.

A reprodução do experimento com amostras representativas das 6 outras regiões do Brasil, proporcionariam resultados nacionais.

\section{3 - VARIÁVEIS LEVANTADAS}

As variáveis responsáveis pelo posicionamento dos sujeitos em posturas de trabalho sentada são:

- Variável I

altura entre piso e assento, tomada do ponto SRP (Syting referential point) ao piso na vertical.

\section{Variável 2}

distância da plataforma de apoio dos pés e assento, tomada da projeção da borda anterior do assento 
ao eixo pivoliador da plataforma na horizontal.

\section{- Variável 3}

angulação entre encosto e assento, tomada a partir do ângulo de $90^{\circ}$, formado pelo encosto e assento no SRP.

\section{Variável 4}

angulação da superfície do assento, tomada da borda anterior ao assento a partir do ângulo de $90^{\circ}$ formado pelo encosto e assento.

\section{- Variável 5}

angulação da plataforma de apoio dos pés e piso, tomada a partir do eixo de pivotiamento da plataforma e a horizontal.

\section{4 - DEFINIÇÃO DA AMOSTRA EXPERIMENTAL}

Com base na altura dos sujeitos levantados pelo IBGE $^{2}$, e utilizando o critério de alocação ótima (alocação de Neyman) segundo BARNETT ${ }^{5}$ teremos uma amostra assim distribuída:

$\begin{array}{ccccc}\text { ALTURA (m) } & \text { PERCENTIS } & \mathrm{N}^{\mathrm{o}} & \text { DE SUJEITOS } \\ 1.59 \text { a } 1,62 & & 3 \text { a } 10 & & 01 \\ 1,62 \text { a } 1,66 & & 10 \text { a } 25 & & 03 \\ 1.66 \text { a } 1.70 & & 25 \text { a } 50 & & 05 \\ 1,70 \text { a } & 1,75 & 50 \text { a } 75 & & 06 \\ 1,75 \text { a } & 1,79 & 75 \text { a } & 90 & 03 \\ 1,79 \text { a } & 1,84 & 90 \text { a } 97 & & 02\end{array}$

Todos os sujeitos testados eram homens, com idade variando de 18 a 40 anos, sem problemas físicos aparentes, com alturas representativas da população. As alturas foram agrupadas por faixa, que correspondem às faixas de percentis da população dos Estados do Paraná, Santa Catarina e Rio Grande do Sul, classificada como região III segundo o IBGE - Instituto Brasileiro de Geografia e Estatística. A população masculina destes estados representam $22 \%$ da PEA - População Economicamente Ativa do Brasil.

\section{5 - EQUIPAMENTO}

O equipamento é constituído de um assento que pode sofrer variações de altura e ângulo, um encosto que também tem seu ângulo de inclinação regulável e uma plataforma de apoio para os pés que pode ser movimentada horizontalmente e posicionada em diferentes ângulos.

O assento é regulado em sua altura de forma contínua por um sistema de elevação mecânica, e a variável 1 altura entre piso e assento é obtida através de uma simples leitura; em um sistema de referencia numérica, posicionado entre estes dois elementos A variável 4 - angulação da Superfície do assento, é regulada de 2,5 em 2,5 graus, através de regulagens discretas, dentro de uma faixa de 0 a 10 graus.

$\mathrm{O}$ encosto tem seu ângulo de inclinação em relação ao assento, regulado de forma discreta, de $5^{\circ}$ em $5^{\circ}$ graus dentro de uma faixa de 90 a 135 graus utravés desta regulagem se obtém a variável 3 .
O movimento horizontal da plataforma de apoio dos pés, cujo curso é de 0 a $550 \mathrm{~mm}$, é possível pela utilização de trilhos laterais e é regulado de forma discreta de $50 \mathrm{em} 50 \mathrm{~mm}$. O posicionamento horizontal da plataforma dá origem à variável 2 .

Os ângulos entre a plataforma de apoio dos pés e piso, variável 5 , são obtidos de forma discreta, de $5 \mathrm{em}$ 5 graus, dentro de uma faixa de 0 a 90 graus.

\section{6 - EXPERIMENTO}

Os 20 sujeitos definidos pela amostra, foram solicitados verbalmente a se posicionarem de forma mais confortável possível, considerando-se sempre o equilíbrio das partes intersegmentares de seu corpo.

As recomendações mais importantes foram de que não deveriam sentir:

1 - Pressão da superfície do assento na parte posterior da coxa;

2 - Que a superfície dos pés ficasse completamente apoiadas na superfície da plataforma de apoio dos pés;

3 - Que a parte posterior do tronco não se deslocasse para frente, e se mantivesse completamente apoiada pelo encosto,

4 - Que ao se regular os ângulos da superfície do assento, isto não provocasse pressões na coxa ou mesmo em suas costas;

5 - Que o ângulo da superfície da plataforma de apoio dos pés, não deveria criar pressões entre o pé a a perna.

Obedecendo o esquema de regulagem das variáveis conforme ilustração foram efetuados os levantamentos obtendo-se os resultados, demonstrados a seguir no Quadro 1.

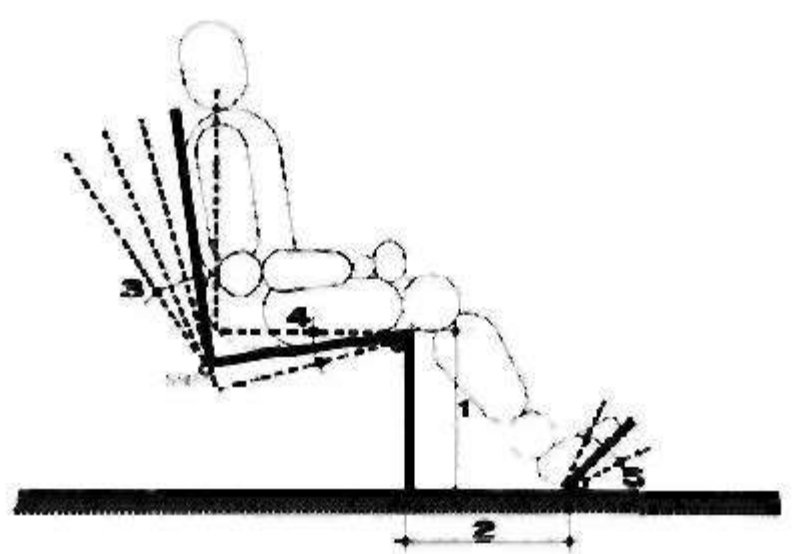



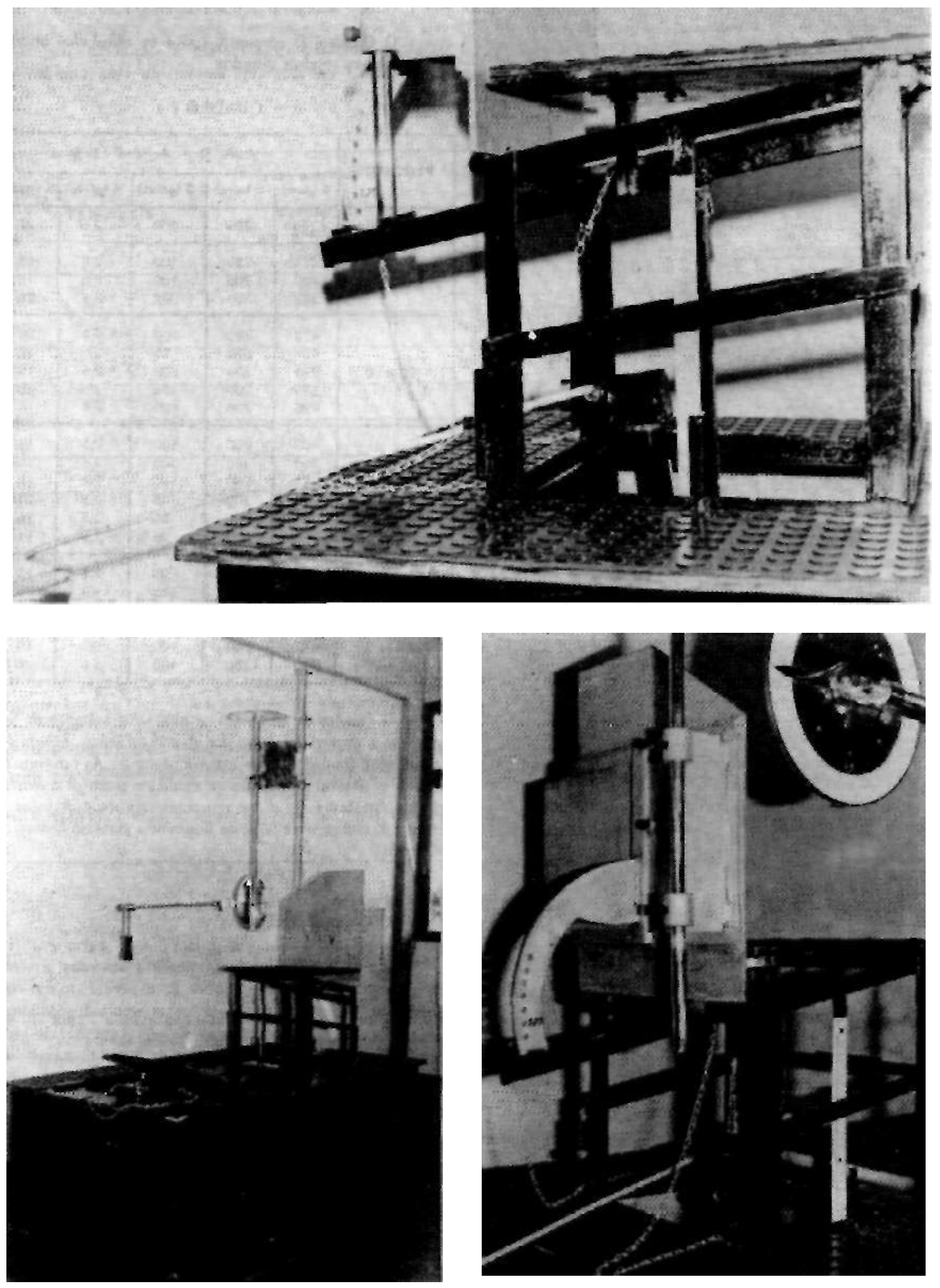

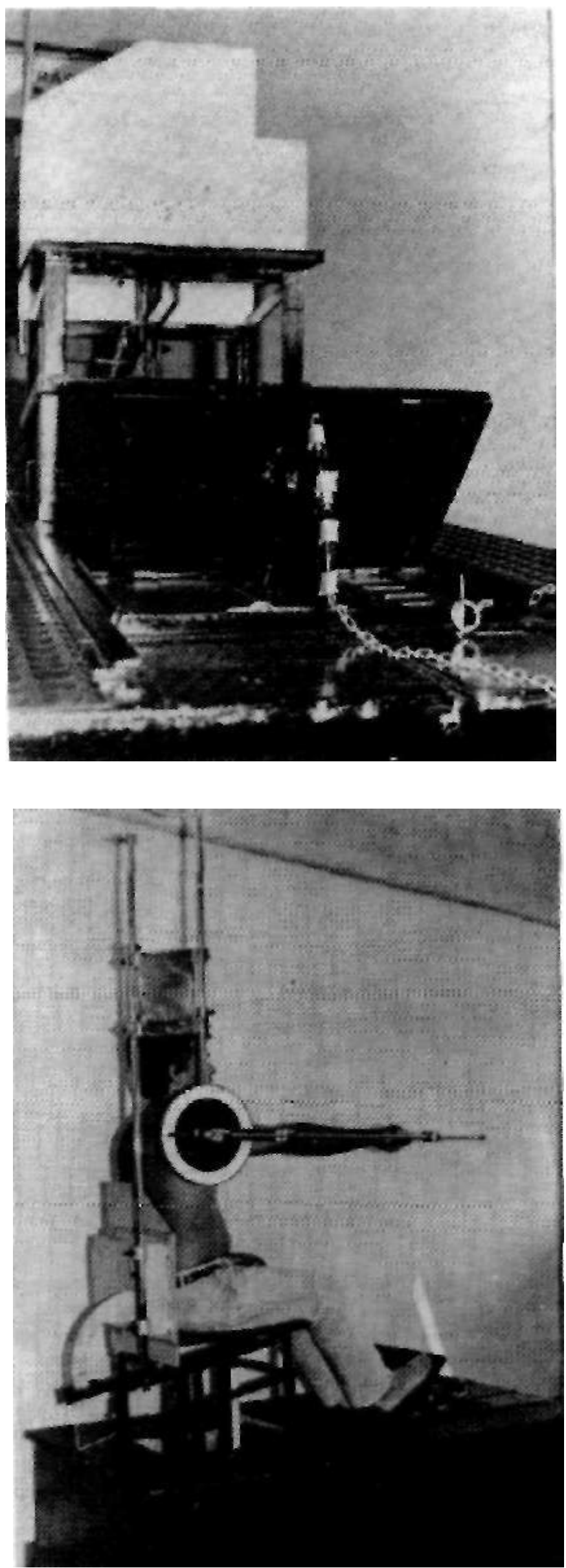

-RESULTADOS

0 Quadro 1, apresenta todos os resultados obtidos com todos os sujeitos testados.

QUADRO 1

\begin{tabular}{|c|c|c|c|c|c|}
\hline \multirow{2}{*}{ PERCENTIS } & \multicolumn{5}{|c|}{$V A R I A V E I E$} \\
\hline & $1(\mathrm{~mm})$ & $2(\mathrm{~mm})$ & 3 (graus) & 4 (errous) & 5 (graus) \\
\hline $3 * 10$ & 370 & 200 & 100 & 2,6 & 20 \\
\hline 70 a 25 & $\begin{array}{l}414 \\
407 \\
305\end{array}$ & $\begin{array}{l}2 b 0 \\
200 \\
300\end{array}$ & $\begin{array}{r}100 \\
100 \\
95\end{array}$ & $\begin{array}{l}2,5 \\
2,5 \\
2,5\end{array}$ & $\begin{array}{l}25 \\
15 \\
25\end{array}$ \\
\hline 25 a 50 & $\begin{array}{l}418 \\
414 \\
390 \\
370 \\
415\end{array}$ & $\begin{array}{l}300 \\
300 \\
250 \\
300 \\
250\end{array}$ & $\begin{array}{r}100 \\
95 \\
100 \\
105 \\
100\end{array}$ & $\begin{array}{l}5,0 \\
2,6 \\
2,5 \\
2.5 \\
2.5\end{array}$ & $\begin{array}{l}30 \\
26 \\
15 \\
90 \\
15\end{array}$ \\
\hline 50 a 76 & $\begin{array}{l}403 \\
390 \\
411 \\
378 \\
435 \\
405\end{array}$ & $\begin{array}{l}250 \\
350 \\
250 \\
200 \\
300 \\
300\end{array}$ & $\begin{array}{r}100 \\
100 \\
100 \\
105 \\
95 \\
95\end{array}$ & $\begin{array}{l}2.5 \\
2.5 \\
2,5 \\
7,5 \\
2,5 \\
2,5\end{array}$ & $\begin{array}{l}15 \\
15 \\
25 \\
25 \\
2 b \\
15\end{array}$ \\
\hline 75 a 90 & $\begin{array}{l}410 \\
413 \\
437\end{array}$ & $\begin{array}{l}350 \\
350 \\
300\end{array}$ & $\begin{array}{r}95 \\
10 b \\
100\end{array}$ & $\begin{array}{l}2.5 \\
b 0 \\
2.5\end{array}$ & $\begin{array}{l}26 \\
25 \\
25\end{array}$ \\
\hline 90 a 97 & $\begin{array}{l}442 \\
440\end{array}$ & $\begin{array}{l}350 \\
360\end{array}$ & $\begin{array}{l}100 \\
100\end{array}$ & $\begin{array}{l}5.0 \\
6.0\end{array}$ & $\begin{array}{l}15 \\
25\end{array}$ \\
\hline
\end{tabular}

O Quadro 2, apresenta os mesmos resultados de forma que a variável 1 é mostrada através da média, obtida dos resultados dos sujeitos do mesmo percentil As variáveis 2 a 5 sob o critério de cálculo de médias e também o resultado da freqüência geral de aparecimento em todo o experimento, indicado na faixa de freqüência geral no Quadro 2. Quadro 2.

8

\section{- CONCLUSÕES}

A definição de variáveis a serem levantadas, a mostra experimental, as recomendações de experimento e o equipamento, indicam que pode-se obter resultados nacionais.

Os custos de construção do equipamento são signi ficativamente baixos, e sua forma de fácil reprodutibilidade. 0 experimento $e ́$ simples de se realizar.

$\mathrm{O}$ maior inconveniente para um levantamento à nível nacional, são as definições das amostras para as demais regiões, uma vez que para tanto, haveria de se obter os coeticientes de variància de cada uma das amostras das outras regiões para o cálculo amostrai, dados estes cujos custos pedido pelo IBGE- Instituto Brasileiro de Geografia e Estatística para fornecê-los, foram significativamente altos. Como este órgão é o único que dispõe destas informações, ficamos impossibilitados de neste trabalho fome- 
cer os dados amostrais para as outras regiões.

A utilização dos dados em princípio, indicam que sejam para definição de dimensões de postos e ou estação de trabalho; para um melhor aproveitamento sugerimos a reprodução do experimento, alocando no equipamento ou nas suas proximidades, todos os elementos que reproduzam a configuração física dos postos ou estações de trabalho.

QUADRO 2

\begin{tabular}{|c|c|c|c|c|c|}
\hline \multirow[b]{2}{*}{ PERCENTIS } & \multirow{2}{*}{$\begin{array}{l}\text { Madła calculada } \\
\text { Variávoi } 1 \\
\text { (mm) } \\
\text { Media }\end{array}$} & \multirow[b]{2}{*}{$\begin{array}{c}\text { Varifugl } 2 \\
\text { (araust } \\
\text { Madia }\end{array}$} & \multicolumn{3}{|c|}{ 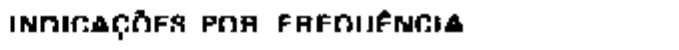 } \\
\hline & & & $\begin{array}{c}\text { Varitgual } 3 \\
\text { \{graus| } \\
\text { Modia }\end{array}$ & $\begin{array}{c}\text { Varibvel } 4 \\
\text { \{graus| } \\
\text { Madia }\end{array}$ & $\begin{array}{c}\text { Varianel } 5 \\
\text { (araus) } \\
\text { Malie }\end{array}$ \\
\hline 3 a 10 & 370 & 200 & 100 & 2,5 & 20 \\
\hline 10 a 25 & 405 & 250 & 98.3 & 2.5 & 21.6 \\
\hline $25+50$ & 401 & 280 & 100 & 3.0 & 10 \\
\hline 50 a 35 & 404 & $2 / b$ & 39,1 & 3,3 & 20 \\
\hline 75090 & 420 & 333,3 & 66,1 & 3,3 & 25 \\
\hline $90 \times 97$ & 441 & 350 & 100 & 5 & 20 \\
\hline $\begin{array}{c}\text { Fraquencia } \\
\text { Geral }\end{array}$ & & $\begin{array}{l}200.3 \\
250.5 \\
300.7 \\
3505\end{array}$ & $\begin{array}{r}96 \cdot 3 \\
100.14 \\
105.3\end{array}$ & 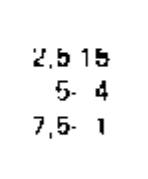 & $\begin{array}{l}10 \cdot 1 \\
16 \\
20 \cdot 1 \\
25 \cdot 10 \\
30 \cdot 1\end{array}$ \\
\hline
\end{tabular}

\section{ABSTRACT}

By regulating the five variables responsible for proper sinting. an experiment was conducred that generated data conceming the best recomendations for the positioning of a sitting subject. A methodology was established for similor in other regions of Brazil, and for the establishing of proper anthropometric parametcrs best suited to the characteristics of the Brasilian population. These parameters should replace foreign data in the design of objects for the work space in Brazil. other experiments based upon these results are indicated.

KEY WORDS: Human engineering; Anthropometry; Workspace; Industrial design; Industrial engineering.

\section{REFERENCIAS BIKIJTKKÁFILAS}

1 - MATIAS, Lorto. Envoltório de alcance - uma experieincia brasileira Rio do Janairo, 1983. Tesa (Mestr, - COPPE/ UFRJ).

2 - IBGE - Institu to Brasileiro de Goografia * Estatistica. Esfudo Nacional da Despesa Familiar. Hio de Janeiro, 1977.

3 - COBlentZ, A, at alii, Utilization O'une Methode de Biostereometrie dans $L$ a Conception d'un Poste de Pilotage d'helicapeterc. OTAN, Paris, (29):1-19, 1975.
4 - IGNAZl, G. at alii. Reconstitution en Trois Dimensions des Airas d'Atteintes du Membre Superieur. Calliers d'Anthropolokie, Paris, |31: 93-117, 1979.

5 BARNETT, V. Elements of Sampling Theory. London, The English Univarsity Press, 1974.

Rectubido pard publiç⿻ุ丷a um 18/03/88 